(1)

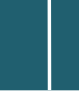

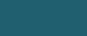

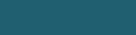

\title{
RESEARCH
}

\section{Association of single-nucleotide polymorphisms in the IL27 gene with autoimmune thyroid diseases}

\author{
Weiwei He' ${ }^{1}$, Bin Wang ${ }^{2}$, Kaida Mu³ , Jing Zhang ${ }^{3}$, Yanping Yang ${ }^{3}$, Wei Yao ${ }^{3}$, Sheli Li ${ }^{1}$ and Jin-an Zhang ${ }^{3}$ \\ 'Department of Endocrinology, Affiliated Hospital of Yanan Medical University, Shaanxi, China \\ 2Department of Endocrinology, Jinshan Hospital of Fudan University, Shanghai, China \\ ${ }^{3}$ Department of Endocrinology, Shanghai University of Medicine \& Health Sciences Affiliated Zhoupu Hospital, Shanghai, China
}

Correspondence should be addressed to S Li or J Zhang: ya116116@126.com or zhangjinan@hotmail.com

\begin{abstract}
Background: Accumulating data have shown that interleukin-27 (IL27) polymorphisms are linked to the susceptibility of some autoimmune diseases. We assessed whether there was an association between three single-nucleotide polymorphisms (SNPS) of IL27 gene and autoimmune thyroid diseases (AITDs).

Methods: Three SNPs (rs153109, rs17855750 and rs181206) of IL27 gene were genotyped by Hi-SNP high-throughput geneotyping in 843 patients with AITDs (516 Graves' disease (GD) and 327 Hashimoto's thyroiditis (HT)) and 677 healthy controls in Chinese Han population.

Results: Compared with controls, rs153109 displayed significant associations with GD in allele and genotype frequencies ( $P=0.002$ and $P=0.008$, respectively) and $\mathrm{rs} 17855750$ displayed significant associations with HT in allele frequencies $(P=0.02)$, whereas no differences in genotype or allele frequencies were found between AITD patients and controls at rs181206.

Conclusion: Our study, for the first time, showed the significant association of the IL27 gene SNPs with AITD.
\end{abstract}

\section{Key Words}

- interleukin-27

- single-nucleotide polymorphism

- autoimmune thyroid diseases

- susceptibility

\section{Introduction}

In autoimmune thyroid diseases (AITDs), one of the autoimmune diseases, cytokine-mediated immunity play a key role during the pathogenesis and development (1). AITDs are common endocrine autoimmune diseases with prevalence of about $5 \%$ in the general population, and iodine intake plays a different role in different regions $(2,3,4,5)$. A recent study in China suggested that the prevalence of AITD was $10.5 \%$ in men and $21.4 \%$ in women (6). It is universally acknowledged that the combined effects of genetic, environmental and immune factors are involved in pathogenesis of AITDs. More and more studies have shown that genetic susceptibility is closely related to AITDs, and may play a key role in the pathogenesis of AITDs and disease progression
$(7,8)$. AITDs mainly have two types of symptoms, Graves' disease (GD) and Hashimoto's thyroiditis (HT). HT is the most common thyroid inflammation characterized by abnormally elevated thyroglobulin antibodies (TGAb) and thyroid peroxidase antibodies (TPOAb). It causes hypothyroidism in the clinics and shows clinical manifestations opposite to GD (9); HT is more common in middle-aged women (10). The cause of HT is still unclear, although family aggregation that often occurs in generations of the same family has been identified in HT. GD is one of the most common causes of hyperthyroidism characterized by positive thyroid-stimulating hormone (TSH) receptor antibodies (TRAb) and is an organspecific autoimmune disease with increased secretion of
This work is licensed under a Creative Commons Attribution-NonCommercial 4.0 International License. ded from Bioscientifica.com at 04/26/2023 11:15:56AM 
thyroid hormone (TH). TRAb can bind to TSH receptor on the thyroid follicular cells and stimulate the production of thyroid hormones (11). GD is more common in women with the ratio of male to female of 1:4-6, particularly in patients in the 20-40 years age group. We have previously found that polymorphisms in some genes such as CTLA4 and PTPN22 $(12,13)$ were associated with GD.

Interleukin (IL)-27 (IL27) has an important role in shaping Th cell responses and is involved in the development of some autoimmune diseases $(14,15)$. IL27 is a member of IL-12/IL-6 family that includes EpsteinBarr virus-induced gene 3 and IL27 p28 subunit, located on chromosome 16p11 (16), which is one of the crucial candidate genes for the development and differentiation of T cells. Nowadays, increasing evidences have confirmed that polymorphisms of IL27 gene play significant roles in some inflammatory diseases and autoimmune diseases, such as inflammatory bowel disease (IBD) (14), rheumatoid arthritis (RA) (17), Behcet's disease (BD) (18) and systemic lupus erythematosus (SLE) (19). A study has shown that IL27 is upregulated in patients with active IBD (20), but not in patients with type 1 diabetes (21). Although IL27 is closely related to many autoimmune inflammatory diseases, the relationship between IL27 and AITD has not been well investigated. Hence, in this study, we performed a controlled case study with a large sample size to investigate the relationship between three IL27 SNPs (rs153109, rs181206 and rs17855750) and AITDs in a Chinese Han population.

\section{Methods}

\section{Patients and controls}

A total of 843 patients with AITDs (516 GD and 327 HT) and 677 healthy controls were enrolled in the study. AITD patients were recruited from Department of Endocrinology, Jinshan Hospital of Fudan University. The inclusion criteria of all patients have been described previously $(12,22)$. Both GD and HT were diagnosed according to the international diagnostic criteria (14). The inclusion criteria for GD were hyperthyroidism, decreased TSH level, toxic diffuse and positive for TRAb. The inclusion criteria for HT were positive for TPOAb or TGAb as well as diffuse hypoechogenicity on thyroid, and with or without hypothyroidism. Common autoimmune diseases were excluded in the controls according to disease history and family history, such as SLE, RA, ankylosing spondylitis, ulcerative colitis (UC), IBD, multiple sclerosis,
Sjogren's syndrome and type 1 diabetes mellitus. The control group had no history of thyroid diseases or other autoimmune diseases. Controls with serious illness or chronic inflammatory diseases were also excluded. The healthy controls matched to the patients by geographic area. The study was approved by the Ethics Committee of Zhoupu Hospital and written informed consent was obtained from all participants.

\section{Genotyping}

A standard protocol with $2 \mathrm{~mL}$ peripheral blood from each participant was used for DNA extraction $(23,24)$. Genomic DNA was extracted from peripheral blood leukocytes using standard procedures of the RelaxGene Blood DNA System (Tiangen Biotech, Beijing, China). Besides, the concentration of DNA samples and the proportion of A260/A280 were measured using the NanoDrop 2000 spectrophotometer (Thermo Scientific Company). The IL27 SNPs were detected using Hi-SNP high-throughput genotyping method (Shanghai Biowing Applied Biotechnology CO. LTD, Shanghai, China). The forward and reverse primers for amplification of rs153109 were 5'-GACTGGGACTGGGACTCAGCAGG-3' and 5'-CATGGCCTCTACAGAGCAGAAACACC-3', respectively. The forward and reverse primers for amplification of rs17855750 were 5'-CAAGCTCTCTCCCTGTCTTG-3' and 5'-TGCATTAGGGGGACTTACAAAG-3, respectively. The forward and reverse primers for amplification of rs181206 were $5^{\prime}$-GGACCTTTCCTGACCATCTCCCTC-3' and 5'-GCAGATCGCGGAGGTCCAG-3', respectively.

\section{Statistical analysis}

The sample size calculation was performed with an expected OR of 1.40. The sample size to make a sufficient power over $80 \%$ required at least 331 cases and 265 controls for rs153109, and at least 799 cases and 640 controls for both rs181206 and rs17855750. The final sample size in our casecontrol study led to a statistical power of over $95 \%$ to detect a significant result. Hardy-Weinberg equilibrium (HWE) was performed using $\chi^{2}$ test. STATA was used in statistical analysis with odds ratios (ORs) and $95 \%$ confidence intervals (95\% CI). Chi-square test was utilized to compare the allele and genotype frequencies between cases and controls. The relationship between IL27 and AITDs was analyzed through multiple comparison models, including allele model, dominant model, recessive model, homozygous model and additive model. Multivariate logistic regression analyses

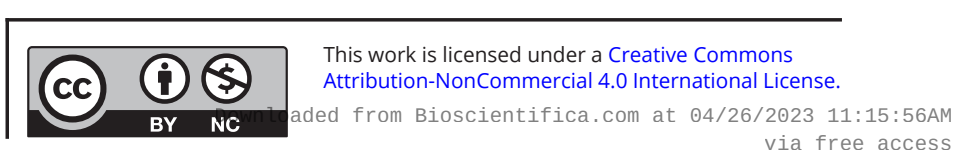


were also performed, and age and gender were used as confounding factors. $P$ value less than 0.05 was considered significant.

\section{Results}

\section{Subject features}

Table 1 shows the baseline data of all participants in this study. There were 843 AITD patients including 516 GD and 327 HT individuals, respectively, and 677 control individuals. Among the GD patients, 357 (69.19\%) were female and 159 (30.81\%) were male. It's worth noting that 106 (20.54\%) GD patients had family history of AITDs and 82 (51.89\%) had ophthalmopathy. In addition, 140 (27.13\%), 239 (46.32\%) and 33 (6.4\%) GD patients had degree I, II and III goiter, respectively, and 104 (20.15\%) GD patients had no goiter. Among the HT patients, $276(84.40 \%)$ were female and $51(15.60 \%)$ were male. In addition, 53 (16.21\%) HT patients had family history of AITDs, 76 (23.24\%), 106 (32.42\%) and 8 (2.44\%) HT patients had degree I, II and III goiter, respectively, and 137 (41.90\%) HT patients had no goiter. Among the 677 healthy individuals, 405 (59.82\%) were female and $272(40.18 \%)$ were male. The average age was $41.82 \pm 14.56$ years old for GD patients, $41.84 \pm 14.52$ years old for HT patients and 39.48 \pm 9.10 years old for healthy controls. There were no significant differences in age and gender among the GD patients, HT patients and healthy controls $(P<0.05)$. No deviation from the HWE was observed for rs153109, rs181206 and rs17855750 in the healthy controls $(P>0.05)$.

\section{Association between rs153109 and AITDs}

The genotyping results for all patients and controls are summarized in Table 2. The genotype frequencies of TT, TC and CC in rs153109 polymorphism were 41.99\%, $46.38 \%$ and $11.63 \%$ in AITD patients and 38.26\%, 47.56\% and $14.18 \%$ in the healthy controls, respectively (Table 2). The genotype frequencies of TT, TC and CC in rs153109 polymorphism were $45.35 \%, 45.35 \%$ and $9.30 \%$ in GD patients and $36.70 \%, 48.01 \%$ and $15.29 \%$ in HT patients, respectively. These results showed that there was no statistically significant difference between AITD patients and healthy controls in both genotype distribution $(P=0.189)$ and allele frequencies of rs153109 $(P=0.073)$. It's worth noting that there were significant differences in the genotype distribution of rs153109 between GD patients and healthy controls $(P=0.008)$ and allele frequencies of rs153109 $(P=0.002)$. By contrast, there were no statistically significant differences in genotype distribution between HT patients and healthy controls $(P=0.844)$ and allele frequencies of rs153109 $(P=0.564)$.

The ORs of the associations of rs153109 of IL27 gene with AITDs before and after adjusting for confounders (age and gender) clearly show that (1) no significant association between rs153109 of IL27 and AITDs was observed under all comparison models (Table 3, P>0.05), (2) no significant association between rs153109 of IL 27

Table 1 Baseline clinical and demographics features of patients enrolled in the study.

\begin{tabular}{|c|c|c|c|}
\hline Items & GD & HT & Controls \\
\hline Number & 516 & 327 & 677 \\
\hline \multicolumn{4}{|l|}{ Gender } \\
\hline Male & $159(30.81 \%)$ & $51(15.60 \%)$ & $272(40.18 \%)$ \\
\hline Female & 357 (69.19\%) & 276 (84.40\%) & 405 (59.82\%) \\
\hline Age (years) & $41.82 \pm 14.56$ & $41.84 \pm 14.52$ & $39.48 \pm 9.10$ \\
\hline \multicolumn{4}{|l|}{ Goiter } \\
\hline No goiter & $104(20.15 \%)$ & 137 (41.90\%) & - \\
\hline Grade I goiter & $140(27.13 \%)$ & $76(23.24 \%)$ & - \\
\hline Grade II goiter & $239(46.32 \%)$ & $106(32.42 \%)$ & - \\
\hline Grade III goiter & $33(6.4 \%)$ & $8(2.44 \%)$ & - \\
\hline \multicolumn{4}{|l|}{ Family history } \\
\hline$(+)$ & $106(20.54 \%)$ & $53(16.21 \%)$ & - \\
\hline$(-)$ & $410(79.46 \%)$ & $274(83.79 \%)$ & - \\
\hline \multicolumn{4}{|l|}{ Ophthalmopathy } \\
\hline$(+)$ & $82(51.89 \%)$ & $3(0.92 \%)$ & - \\
\hline$(-)$ & $434(84.11 \%)$ & $324(99.08 \%)$ & - \\
\hline
\end{tabular}

GD, Graves' disease; HT, Hashimoto's thyroiditis.

https://ec.bioscientifica.com https://doi.org/10.1530/EC-18-0370
(C) 2019 The authors Published by Bioscientifica Ltd

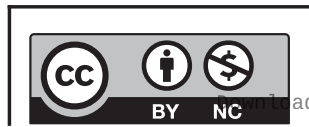

This work is licensed under a Creative Commons Attribution-NonCommercial 4.0 International License. ded from Bioscientifica.com at 04/26/2023 11:15:56AM via free access 
Table 2 Allele frequencies and genotype distribution of IL-27 polymorphisms in AITD patients and healthy controls.

\begin{tabular}{l}
\hline Gene/SNP \\
\hline IL27 \\
\hline rs153109 \\
T \\
C \\
TT \\
TC \\
CC \\
rs181206 \\
T \\
C \\
TT \\
TC \\
CC \\
rS17855750 \\
T \\
G \\
TT \\
TG \\
GG \\
\hline
\end{tabular}

\begin{tabular}{c}
\hline Controls \\
\hline$n(\%)$ \\
\hline
\end{tabular}

$840(62.04)$

$514(37.96)$

$259(38.26)$

$322(47.56)$

$96(14.18)$

$$
\begin{gathered}
1233(91.06) \\
121(8.94) \\
564(83.31) \\
105(15.51) \\
8(1.18)
\end{gathered}
$$

\begin{tabular}{c}
$1230(90.84)$ \\
$124(9.16)$ \\
$560(82.72)$ \\
$110(16.25)$ \\
$7(1.03)$ \\
\hline
\end{tabular}

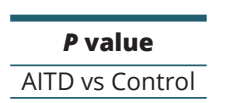

$1099(56.18)$

587 (34.82)

354 (41.99)

391 (46.38)

$98(11.63)$

$$
\begin{gathered}
1545(91.64) \\
141(8.36) \\
707(83.87) \\
131(15.54) \\
5(0.59)
\end{gathered}
$$

1501 (89.03)

185 (10.97)

665 (78.89)

$171(20.28)$

$7(0.83)$

\begin{tabular}{|c|c|c|c|}
\hline GD & $P$ value & HT & $P$ value \\
\hline$n(\%)$ & GD vs Control & $n(\%)$ & HT vs Control \\
\hline 702 (68.02) & 0.002 & $397(60.70)$ & 0.564 \\
\hline 330 (31.98) & & $257(39.30)$ & \\
\hline 234 (45.35) & 0.008 & 120 (36.70) & 0.844 \\
\hline 234 (45.35) & & $157(48.01)$ & \\
\hline 48 (9.30) & & 50 (15.29) & \\
\hline 946 (91.67) & 0.604 & 599 (91.59) & 0.696 \\
\hline $86(8.33)$ & & 55 (8.41) & \\
\hline 432 (83.72) & 0.327 & $275(84.10)$ & 0.906 \\
\hline $82(15.89)$ & & 49 (14.98) & \\
\hline $2(0.39)$ & & $3(0.92)$ & \\
\hline 327 & & & \\
\hline 928 (89.92) & 0.449 & 573 (87.61) & 0.02 \\
\hline $104(10.08)$ & & 81 (12.39) & \\
\hline 413 (80.04) & 0.068 & $252(77.06)$ & 0.087 \\
\hline 102 (19.77) & & $69(21.10)$ & \\
\hline 1 (0.19) & & $6(1.84)$ & \\
\hline
\end{tabular}

AITD, autoimmune diseases; GD, Graves' disease; HT, Hashimoto's thyroiditis.

gene and AITDs was observed after adjustment for age and gender (Table 3), (3) a significant association of IL27 rs153109 with GD was observed under the allele model ( $\mathrm{OR}=0.76,95 \% \mathrm{CI} 0.64-0.91, P=0.002)$, dominant model (OR=0.75, 95\% CI 0.59-0.94; $P=0.014$ ), recessive model ( $\mathrm{OR}=0.62,95 \% \mathrm{CI} 0.43-0.90, P=0.011)$ and homozygous model (OR=0.74, 95\% CI 0.61-0.90, $P=0.003$ ) (Table 4) and (4) no significant association between rs153109 of
IL27 gene and HT was observed under all comparison

\begin{tabular}{|c|c|c|c|c|}
\hline \multirow[b]{2}{*}{ Comparison models } & \multicolumn{2}{|c|}{ Unadjusted estimates } & \multicolumn{2}{|c|}{ Adjusted estimates $^{a}$} \\
\hline & OR $(95 \% \mathrm{Cl})$ & $P$ values & OR $(95 \% \mathrm{Cl})$ & $P$ values \\
\hline \multicolumn{5}{|l|}{ rs153109 } \\
\hline Allele model & $0.87(0.75-1.01)$ & 0.071 & $0.86(0.74-1.00)$ & 0.053 \\
\hline Dominant model & $0.86(0.70-1.05)$ & 0.140 & $0.84(0.68-1.03)$ & 0.097 \\
\hline Recessive model & $0.80(0.59-1.08)$ & 0.138 & $0.79(0.58-1.08)$ & 0.140 \\
\hline Homozygous model & $0.86(0.74-1.02)$ & 0.077 & $0.85(0.72-1.01)$ & 0.063 \\
\hline Additive model & $0.89(0.71-1.10)$ & 0.287 & 0.87 (0.69-1.08) & 0.203 \\
\hline \multicolumn{5}{|l|}{ rs181206 } \\
\hline Allele model & $0.93(0.72-1.20)$ & 0.578 & $0.91(0.71-1.18)$ & 0.487 \\
\hline Dominant model & $0.96(0.73-1.26)$ & 0.770 & $0.94(0.71-1.24)$ & 0.640 \\
\hline Recessive model & $0.50(0.16-1.53)$ & 0.225 & $0.52(0.16-1.63)$ & 0.260 \\
\hline Homozygous model & $0.71(0.40-1.24)$ & 0.224 & $0.72(0.41-1.27)$ & 0.255 \\
\hline Additive model & $1.00(0.75-1.32)$ & 0.973 & $0.97(0.73-1.28)$ & 0.817 \\
\hline \multicolumn{5}{|l|}{ rs17855750 } \\
\hline Allele model & $1.23(0.96-1.56)$ & 0.098 & $1.22(0.96-1.56)$ & 0.111 \\
\hline Dominant model & $1.28(0.99-1.66)$ & 0.061 & $1.28(0.98-1.66)$ & 0.070 \\
\hline Recessive model & $0.80(0.28-2.30)$ & 0.680 & $0.80(0.27-2.35)$ & 0.682 \\
\hline Homozygous model & $0.92(0.54-1.55)$ & 0.749 & $0.91(0.53-1.57$ & 0.743 \\
\hline Additive model & $1.31(1.00-1.71)$ & 0.046 & $1.30(1.00-1.71)$ & 0.054 \\
\hline
\end{tabular}
models (Table 5, $P>0.05$ ). Multivariate logistic regression analyses indicate that rs153109 of IL27 gene is significantly associated with GD (Table 4), but not with AITDs and HT (Tables 3 and 5). Subgroup analysis in GD patients with ophthalmopathy suggested that there was an obvious difference in the genotype distribution and allele frequencies of rs153109 between GD patients

Table 3 Odds ratios (ORs) of the associations of three polymorphisms in IL27 gene with AITDs before and after adjusting for confounders (age and gender).

${ }^{a} \mathrm{Age}$ and gender were adjusted in the multivariate logistic regression analyses. $95 \% \mathrm{CI}, 95 \%$ confidence interval; AITD, autoimmune diseases; OR, odds ratio. 
Table 4 Odds ratios (ORs) of the associations of three polymorphisms in IL27 gene with GD before and after adjusting for confounders (age and gender).

\begin{tabular}{|c|c|c|c|c|}
\hline \multirow[b]{2}{*}{ Comparison models } & \multicolumn{2}{|c|}{ Unadjusted estimates } & \multicolumn{2}{|c|}{ Adjusted estimates ${ }^{a}$} \\
\hline & OR $(95 \% \mathrm{Cl})$ & $P$ values & OR $(95 \% \mathrm{Cl})$ & $P$ values \\
\hline \multicolumn{5}{|l|}{ rs153109 } \\
\hline Allele model & $0.76(0.64-0.91)$ & 0.002 & $0.76(0.64-0.90)$ & 0.002 \\
\hline Dominant model & $0.75(0.59-0.94)$ & 0.014 & $0.74(0.58-0.93)$ & 0.011 \\
\hline Recessive model & $0.62(0.43-0.90)$ & 0.011 & $0.62(0.43-0.90)$ & 0.012 \\
\hline Homozygous model & $0.74(0.61-0.90)$ & 0.003 & $0.74(0.61-0.90)$ & 0.003 \\
\hline Additive model & $0.80(0.63-1.03)$ & 0.080 & $0.79(0.62-1.01)$ & 0.061 \\
\hline \multicolumn{5}{|l|}{ rs181206 } \\
\hline Allele model & $0.93(0.70-1.24)$ & 0.606 & $0.91(0.68-1.22)$ & 0.537 \\
\hline Dominant model & $0.97(0.71-1.32)$ & 0.849 & $0.95(0.70-1.30)$ & 0.751 \\
\hline Recessive model & $0.33(0.69-1.54)$ & 0.157 & $0.34(0.07-1.61)$ & 0.174 \\
\hline Homozygous model & $0.57(0.26-1.24)$ & 0.158 & $0.58(0.27-1.27)$ & 0.171 \\
\hline Additive model & $1.02(0.74-1.40)$ & 0.904 & $1.00(0.73-1.37)$ & 0.981 \\
\hline \multicolumn{5}{|l|}{ rs17855750 } \\
\hline Allele model & $1.12(0.84-1.47)$ & 0.443 & $1.12(0.85-1.48)$ & 0.429 \\
\hline Dominant model & $1.19(0.89-1.60)$ & 0.237 & $1.19(0.89-1.61)$ & 0.241 \\
\hline Recessive model & $0.19(0.02-1.52)$ & 0.116 & $0.21(0.02-1.69)$ & 0.141 \\
\hline Homozygous model & $0.44(0.15-1.26)$ & 0.125 & $0.46(0.16-1.33)$ & 0.155 \\
\hline Additive model & $1.26(0.93-1.69)$ & 0.132 & $1.25(0.93-1.70)$ & 0.141 \\
\hline
\end{tabular}

${ }^{a}$ Age and gender were adjusted in the multivariate logistic regression analyses.

$95 \% \mathrm{Cl}, 95 \%$ confidence interval; GD, Graves' disease; OR, odds ratio.

without ophthalmopathy and controls $(P=0.007$ and $P=0.004$ respectively; Supplementary Table 1 , see section on supplementary data given at the end of this article). Subgroup analysis by gender also found that rs153109 was significantly associated with GD in females for both the genotype distribution $(P=0.024)$ and the allele frequencies $(P=0.007)$ (Supplementary Tables 2 and 3).

\section{Association between rs181206 and AITD}

It is clear from Table 2 that there was (1) no significant difference between AITD patients and healthy controls in both genotype distribution $(P=0.464)$ and allele frequency of IL27 rs181206 ( $P=0.575)$, (2) no significant difference between GD patients and healthy controls

Table 5 Odds ratios (ORs) of the associations of three polymorphisms in IL27 gene with HT before and after adjusting for confounders (age and gender).

\begin{tabular}{l} 
Comparison models \\
\hline rs153109 \\
Allele model \\
Dominant model \\
Recessive model \\
Homozygous model \\
Additive model \\
rs181206 \\
Allele model \\
Dominant model \\
Recessive model \\
Homozygous model \\
Additive model \\
rs17855750 \\
Allele model \\
Dominant model \\
Recessive model \\
Homozygous model \\
Additive model
\end{tabular}

\begin{tabular}{|c|c|}
\hline \multicolumn{2}{|c|}{ Unadjusted estimates } \\
\hline OR $(95 \% \mathrm{Cl})$ & $P$ values \\
\hline $1.06(0.87-1.28)$ & 0.563 \\
\hline $1.07(0.81-1.40)$ & 0.633 \\
\hline $1.09(0.75-1.58)$ & 0.640 \\
\hline $1.06(0.87-1.30)$ & 0.571 \\
\hline $1.05(0.79-1.40)$ & 0.729 \\
\hline $0.94(0.68-1.30)$ & 0.702 \\
\hline $0.94(0.66-1.35)$ & 0.752 \\
\hline $0.77(0.20-2.94)$ & 0.707 \\
\hline $0.88(0.45-1.71)$ & 0.700 \\
\hline $0.96(0.66-1.38)$ & 0.816 \\
\hline 1.39 (1.04-1.87) & 0.028 \\
\hline 1.42 (1.03-1.97) & 0.033 \\
\hline $1.79(0.60-5.37)$ & 0.299 \\
\hline $1.38(0.80-2.40)$ & 0.251 \\
\hline 1.40 (1.00-1.95) & 0.052 \\
\hline
\end{tabular}

\begin{tabular}{|c|c|}
\hline \multicolumn{2}{|c|}{ Adjusted estimates $^{a}$} \\
\hline OR $(95 \% \mathrm{Cl})$ & $P$ values \\
\hline $1.04(0.85-1.28)$ & 0.681 \\
\hline $1.04(0.78-1.39)$ & 0.777 \\
\hline $1.18(0.74-1.60)$ & 0.684 \\
\hline $1.05(0.85-1.29)$ & 0.664 \\
\hline 1.03 (0.76-139) & 0.842 \\
\hline $0.95(0.68-1.34)$ & 0.780 \\
\hline $0.95(0.66-1.38)$ & 0.807 \\
\hline $0.85(0.21-3.40)$ & 0.818 \\
\hline $0.92(0.64-1.84)$ & 0.814 \\
\hline $0.96(0.66-1.41)$ & 0.84 \\
\hline $1.37(1.01-1.87)$ & 0.046 \\
\hline 1.39 (0.99-1.95) & 0.059 \\
\hline $1.95(0.60-6.34)$ & 0.269 \\
\hline $1.43(0.79-2.60)$ & 0.236 \\
\hline $1.35(0.95-1.91)$ & 0.093 \\
\hline
\end{tabular}

${ }^{\mathrm{a}} \mathrm{Age}$ and gender were adjusted in the multivariate logistic regression analyses. 95\% Cl, 95\% confidence interval; HT, Hashimoto's thyroiditis; OR, odds ratio. 
in both the genotype distribution $(P=0.327)$ and allele frequencies of rs181206 ( $P=0.604)$ and (3) no significant difference between HT patients and healthy controls in the genotype distribution $(P=0.906)$ and the allele frequency of rs181206 $(P=0.696)$.

Table 3 shows no significant association of IL27 rs181206 with AITDs under all comparison models before and after adjustment for age and gender $(P>0.05)$. Tables 4 and 5 show no association between IL27 rs181206 and GD or HT under all comparison models $(P>0.05)$.

\section{Association between rs17855750 and AITD}

Table 2 shows that the allele frequencies of $\mathrm{T}$ and $\mathrm{G}$ in rs 17855750 polymorphism were $89.03 \%$ and $10.97 \%$ in AITD patients, $90.84 \%$ and $9.16 \%$ in healthy controls, $89.92 \%$ and $10.08 \%$ in GD patients, and $87.61 \%$ and $12.39 \%$ in HT patients, respectively (Table 2). Our results show that the allele frequencies of rs 17855750 were statistically and significantly different between HT patients and healthy controls $(P=0.02)$.

A mild association of IL27 rs17855750 with AITDs was observed in additive model before adjustment for age and gender (Table 3, $P=0.046$ ). But there was no significant difference in both genotype distribution and allele frequencies of rs17855750 between GD patients and healthy controls (Table 4, $P>0.05$ ). However, as shown in Table 5, a significant association of IL27 rs17855750 with HT was observed under the allele model $(\mathrm{OR}=1.39,95 \% \mathrm{CI}$ 1.04-1.87, $P=0.028)$ and dominant model ( $\mathrm{OR}=1.42,95 \%$ CI 1.03-1.97; $P=0.033$ ). Multivariate logistic regression analyses show that the association was still significant after adjustment for age and gender in allele model $(\mathrm{OR}=1.37$, 95\% CI 1.01-1.87; $P=0.046$ ) (Table 5). In addition, Table 5 shows a slight but not significant association between rs17855750 of IL27 and HT in additive model before adjustment $\quad(\mathrm{OR}=1.40 ; \quad 95 \%$ CI $1.00-1.95 ; \quad P=0.052)$. Subgroup analysis by gender found that rs17855750 was mildly associated with HT in females for both the genotype distribution $(P=0.042)$ and allele frequencies $(P=0.023)$, which suggested that rs17855750 was possibly involved in the pathogenesis of HT, but the association in males was insignificant (Supplementary Tables 2 and 3). Outcomes of subgroup analyses stratified by family history and goiter degree are shown in the Supplementary Tables 4, 5, 6 and 7.

\section{Discussion}

Our study explored the association of three SNPs of IL27 in autoimmune thyroid disease and found that both genotype frequency and allele frequency of rs153109 has a strong correlation with GD. In addition, rs17855750 has a correlation with HT in both allele and dominant models, while rs181206 has no significant positive relationship with GD and HT.

To the best of our knowledge, the present study is the first on the association of IL27 polymorphism with GD predisposition. With the advancement of modern medicine, although the pathogenesis of GD has gradually surfaced, due to its complexity, it has not yet been accurately determined. It is generally believed that interactions between environment and genes play a crucial role in the pathogenesis of GD. Although many genes have been reported to be associated with the susceptibility of GD, there is no well-recognized report to date. The polymorphisms of IL27 gene has been extensively studied in many autoimmune diseases on account of IL27, which is a critical cytokine that functions as a mediator between the innate and adaptive immune system (25). As we all know, GD is closely related to the abnormal activation of $\mathrm{T}$ lymphocytes, and IL27 is a proinflammatory cytokine that plays an essential role in transcriptional activation and regulation of T lymphocytes (26). IL27, a special IL-12/IL-6 family cytokine, is a heterodimeric cytokine mainly produced by antigen-presenting cells, including DCs and monocytes/ macrophages (27). IL27 is a pleiotropic cytokine that has multiple roles in immune function. IL27 has significant antitumor activity because it enhances antitumor immunity by regulating NK cell activity, with strong direct anti-antigen and anti-metastatic effects (28), and may play a key role in the antitumor immune response by regulating the production of T helper 1 (Th1)/Th2 bias and regulatory T cells (Treg) (29). IL27 induces cytotoxic $\mathrm{T}$ lymphocyte production by inducing transcription factor T-bet and cytotoxic effect granzyme B and perforin expression (30). A previous study pointed out that IL-27Rdeficient mice develop profound or fatal T-cell-mediated pathology without modulating IL27 signaling (31). IL27 was viewed as a proinflammatory cytokine before, due to its support to the development of interferon (IFN)secreting T helper cells (Th). However, IL27 has recently been shown to act as a negative regulator of ongoing immune responses during infection and autoimmune inflammation because it can limit the production of proinflammatory cytokines by CD $4 \mathrm{~T}$ cells including IFN and resist the role of IL-6 (32). Therefore, we examined the association of IL27 gene with several autoimmune and inflammatory diseases, analyzed the contribution of three potentially functional SNPs of IL27 and demonstrated a

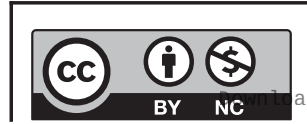

This work is licensed under a Creative Commons Attribution-NonCommercial 4.0 International License. ded from Bioscientifica.com at 04/26/2023 11:15:56AM 
clear association between IL27 polymorphisms and GD susceptibility.

Previous studies showed that rs153109, rs17855750 and rs181206 of IL27 gene displayed significant associations with dilated cardiomyopathy (DCM) (33), atrial fibrillation (AF) (33), allergic rhinitis (34), preeclampsia (35) and renal cell carcinoma (RCC) (36). Thyroid autoimmunity is frequently related to polyglandular endocrine syndromes $(37,38)$. The number of AITD patients with autoimmune polyglandular syndromes in our study was not very common, and the information above was not recorded in detail, so the relationship between autoimmune polyglandular syndromes and IL27 SNPs could not be analyzed. Currently, there are no studies on the relationship between autoimmune polyglandular syndromes and IL27 polymorphisms, and more studies are needed to explore the relationship between them in the future. Existing studies reported that rs153109 AG and AG/GG genotypes, rs17855750 GT and GT/GG genotypes of IL27 gene significantly increased the risk of PTC, both rs153109 and rs17855750 of IL27 gene contributed to the increased risk of PTC (22). Moreover, there is another study which showed that rs153109 of IL27 gene may be associated with the decreased risk for lymph node metastasis of PTC (39). In our present study, rs153109 locus shows a strong correlation with GD and rs17855750 locus shows a significant correlation with HT, but there is no significant association between rs181206 and AITDs including GD and HT.

We have previously shown that thyroid-stimulating hormone receptor (TSHR) (40), CD40 (13), microRNA (23) and IL17 (41) are associated with the pathogenesis of GD and found that polymorphisms of PTPN22 and CTLA4 are associated with the pathogenesis of GD, suggesting that genetic polymorphism plays a key role in the pathogenesis and progression of GD $(12,13)$. Signal transducer and activator of transcription 3 (STAT3) rs3816769 and rs744166 have been shown to be associated with AITD risk and thyroid antibody levels in the Polish population (42). In this study, we further demonstrate a clear association between polymorphism of IL27 and AITDs, and provide genetics evidence for the role of IL27 in the pathogenesis of GD. Though our study indicated IL27 rs153109 locus is a susceptible factor to GD and rs17855750 locus is a susceptible factor to HT, the molecular mechanisms underlying the roles of IL27 rs153109 and IL27 rs17855750 in autoimmune diseases are still unclear. Moreover, IL-23/IL-17 axis plays a crucial role in the pathogenesis of inflammatory and autoimmune diseases (43). There is a study which suggested that the rs2275913 of the
IL17/IL23R pathway is associated with rheumatic heart disease (RHD) in South Indian populations (44). The associations found in the rs11209026 of IL23R and the rs187238 of IL18 genes suggest that a genetically determined high activity of the IL23/IL17 pathway was associated with increased risk of ankylosing spondylitis (45). Yu et al. confirmed the association of SNPs of IL23R and IL17A with UC risk in Chinese Han population (46).

Although our study confirms that IL27 has a significant association with GD, there are still limitations in this study. First, our study found for the first time that the genetic susceptibility of IL27 is clearly associated with GD in Asian populations, but whether it is also true in other ethnic groups needs to be further explored with large sample size. Second, this study only evaluated the association between IL27 gene SNPs and GD susceptibility, and did not analyze the role of IL27 gene SNPs in predicting treatment outcomes. Third, our study tested only three potential SNP sites of IL27 in GD patients and healthy controls. The association of other SNP sites with GD needs to be further evaluated.

In conclusion, this is the first identification of the association of IL27 rs153109 with GD and rs17855750 with HT. Our findings add new data on the genetic contribution to GD susceptibility and support the crucial role of IL27 in GD pathogenesis. However, further researches are warranted to elucidate the molecular mechanisms underlying the relationship between IL27 and GD.

\section{Supplementary data}

This is linked to the online version of the paper at https://doi.org/10.1530/ EC-18-0370.

\section{Declaration of interest}

The authors declare that there is no conflict of interest that could be perceived as prejudicing the impartiality of the research reported.

\section{Funding}

The present work was supported by grants from the National Natural Science Foundation of China (No. 81670722 and 81471004).

\section{References}

1 Caturegli P, Kimura H, Rocchi R \& Rose NR. Autoimmune thyroid diseases. Current Opinion in Rheumatology 200719 44-48. (https://doi. org/10.1097/BOR.0b013e3280113d1a)

2 Valdes S, Maldonado-Araque C, Lago-Sampedro A, Lillo JA, GarciaFuentes E, Perez-Valero V, Gutierrez-Repiso C, Ocon-Sanchez P, Goday A, Urrutia I, et al. Population-based national prevalence of 
thyroid dysfunction in Spain and associated factors: diabetes study. Thyroid 201727 156-166. (https://doi.org/10.1089/thy.2016.0353)

3 Khattak RM, Ittermann T, Nauck M, Below H \& Volzke H. Monitoring the prevalence of thyroid disorders in the adult population of Northeast Germany. Population Health Metrics 2016 14 39. (https:// doi.org/10.1186/s12963-016-0111-3)

4 Shan Z, Chen L, Lian X, Liu C, Shi B, Shi L, Tong N, Wang S, Weng J, Zhao J, et al. Iodine status and prevalence of thyroid disorders after introduction of mandatory universal salt iodization for 16 years in China: a cross-sectional study in 10 cities. Thyroid 201626 1125-1130. (https://doi.org/10.1089/thy.2015.0613)

5 McLeod DS, Cooper DS, Ladenson PW, Whiteman DC \& Jordan SJ Race/Ethnicity and the prevalence of thyrotoxicosis in young Americans. Thyroid 201525 621-628. (https://doi.org/10.1089/ thy.2014.0504)

6 Chen C, Xu H, Chen Y, Chen Y, Li Q, Hu J, Liang W, Cheng J, Xia F, Wang $\mathrm{C}$, et al. Iodized salt intake and its association with urinary iodine, thyroid peroxidase antibodies, and thyroglobulin antibodies among urban Chinese. Thyroid 201727 1566-1573. (https://doi. org/10.1089/thy.2017.0385)

7 Tomer Y. Mechanisms of autoimmune thyroid diseases: from genetics to epigenetics. Annual Review of Pathology 20149 147-156. (https:// doi.org/10.1146/annurev-pathol-012513-104713)

8 Lee HJ, Li CW, Hammerstad SS, Stefan M \& Tomer Y. Immunogenetics of autoimmune thyroid diseases: a comprehensive review. Journal of Autoimmunity 201564 82-90. (https://doi. org/10.1016/j.jaut.2015.07.009)

9 Brix TH, Kyvik KO, Christensen K \& Hegedus L. Evidence for a major role of heredity in Graves' disease: a population-based study of two Danish twin cohorts. Journal of Clinical Endocrinology and Metabolism 200186 930-934. (https://doi.org/10.1210/jcem.86.2.7242)

10 Ajjan RA \& Weetman AP. The pathogenesis of Hashimoto's thyroiditis: further developments in our understanding. Hormone and Metabolic Research 201547 702-710. (https://doi. org/10.1055/s-0035-1548832)

11 Burch HB \& Cooper DS. Management of Graves disease: a review. JAMA 2015314 2544-2554. (https://doi.org/10.1001/ jama.2015.16535)

12 Heward JM, Brand OJ, Barrett JC, Carr-Smith JD, Franklyn JA \& Gough SC. Association of PTPN22 haplotypes with Graves' disease. Journal of Clinical Endocrinology and Metabolism 200792 685-690. (https://doi.org/10.1210/jc.2006-2064)

13 Yang J, Qin Q, Yan N, Zhu YF, Li C, Yang XJ, Wang X, Pandey M, Hou P \& Zhang JA. CD40 C/T(-1) and CTLA-4 A/G(49) SNPs are associated with autoimmune thyroid diseases in the Chinese population. Endocrine 201241 111-115. (https://doi.org/10.1007/ s12020-011-9510-1)

14 Li CS, Zhang Q, Lee KJ, Cho SW, Lee KM, Hahm KB, Choi SC, Yun KJ, Chung HT \& Chae SC. Interleukin-27 polymorphisms are associated with inflammatory bowel diseases in a Korean population. Journal of Gastroenterology and Hepatology 200924 1692-1696. (https://doi. org/10.1111/j.1440-1746.2009.05901.x)

15 Shen H, Xia L, Xiao W \& Lu J. Increased levels of interleukin-27 in patients with rheumatoid arthritis. Arthritis and Rheumatism 201163 860-861. (https://doi.org/10.1002/art.30180)

16 Pflanz S, Timans JC, Cheung J, Rosales R, Kanzler H, Gilbert J, Hibbert L, Churakova T, Travis M, Vaisberg E, et al. IL-27, a heterodimeric cytokine composed of EBI3 and p28 protein, induces proliferation of naive CD4+ T cells. Immunity 200216 779-790. (https://doi.org/10.1016/S1074-7613(02)00324-2)

17 Paradowska-Gorycka A, Raszkiewicz B, Jurkowska M, Felis-Giemza A Romanowska-Prochnicka K, Manczak M \& Olesinska M. Association of single nucleotide polymorphisms in the IL27 gene with rheumatoid arthritis. Scandinavian Journal of Immunology $2014 \mathbf{8 0}$ 298-305. (https://doi.org/10.1111/sji.12209)
18 Dehghanzadeh R, Babaloo Z, Sakhinia E, Khabazi A, Shanehbandi D, Sadigh-Eteghad S \& Gharibi T. IL-27 gene polymorphisms in Iranian patients with Behcet's disease. Clinical Laboratory 201662 855-861. (https://doi.org/10.7754/Clin.Lab.2015.150843)

19 Paradowska-Gorycka A, Sowinska A, Stypinska B, Grobelna MK, Walczyk M, Olesinska M, Piotrowski P \& Jagodzinski PP. Genetic variants in IL-12B and IL-27 in the polish patients with systemic lupus erythematosus. Scandinavian Journal of Immunology $2016 \mathbf{8 4}$ 49-60. (https://doi.org/10.1111/sji.12439)

20 Furuzawa Carballeda J, Fonseca Camarillo G \& YamamotoFurusho JK. Interleukin 27 is up-regulated in patients with active inflammatory bowel disease. Immunologic Research 201664 901-907. (https://doi.org/10.1007/s12026-016-8804-z)

21 Santos AS, Melo ME, Crisostomo LG, Fukui RT, Matioli SR \& Silva ME. Lack of association between IL27 gene variants and type 1 diabetes susceptibility. Cytokine 201361 349-352. (https://doi. org/10.1016/j.cyto.2012.12.003)

22 Nie X, Yuan F, Chen P, Pu Y, Zhu J, Wang Y, Xiao X, Che G, Gao L \& Zhang L. Association between IL-27 gene polymorphisms and risk of papillary thyroid carcinoma. Biomarkers in Medicine 201711 141-149. (https://doi.org/10.2217/bmm-2016-0283)

23 Cai T, Li J, An X, Yan N, Li D, Jiang Y, Wang W, Shi L, Qin Q, Song R, et al. Polymorphisms in MIR499A and MIR125A gene are associated with autoimmune thyroid diseases. Molecular and Cellular Endocrinology 2017440 106-115. (https://doi.org/10.1016/j. mce.2016.11.017)

24 Song RH, Qin Q, Yan N, Muhali FS, Meng S, He ST \& Zhang JA. Variants in IRAK1-MECP2 region confer susceptibility to autoimmune thyroid diseases. Molecular and Cellular Endocrinology 2015399 244-249. (https://doi.org/10.1016/j.mce.2014.10.013)

25 Wynick C, Petes C \& Gee K. Interleukin-27 mediates inflammation during chronic disease. Journal of Interferon and Cytokine Research 201434 741-749. (https://doi.org/10.1089/jir.2013.0154)

26 Ouaked N, Mantel PY, Bassin C, Burgler S, Siegmund K, Akdis CA \& Schmidt-Weber CB. Regulation of the foxp3 gene by the Th1 cytokines: the role of IL-27-induced STAT1. Journal of Immunology 2009182 1041-1049. (https://doi.org/10.4049/ jimmunol.182.2.1041)

27 Jung JY, Roberts LL \& Robinson CM. The presence of interleukin-27 during monocyte-derived dendritic cell differentiation promotes improved antigen processing and stimulation of T cells. Immunology 2015144 649-660. (https://doi.org/10.1111/imm.12417)

28 Pontrelli P, Rascio F, Zaza G, Accetturo M, Simone S, Infante B, Furian L, Castellano G, Ditonno P, Battaglia M, et al. Interleukin-27 is a potential marker for the onset of post-transplant malignancies. Nephrology Dialysis Transplantation 201934 157-166. (https://doi. org/10.1093/ndt/gfy206)

29 Hasegawa H, Mizoguchi I, Chiba Y, Ohashi M, Xu M \& Yoshimoto T. Expanding diversity in molecular structures and functions of the IL-6/IL-12 heterodimeric cytokine family. Frontiers in Immunology 20167 479. (https://doi.org/10.3389/fimmu.2016.00479)

30 Zhou H, Qiu J-h, Wang T, Yu Y-y, Liu X-n, Li X, Wang Y-w, Hou Y, Li L-z, Liu X-g, et al. Interleukin 27 inhibits cytotoxic T-lymphocyte-mediated platelet destruction in primary immune thrombocytopenia. Blood 2014124 3316-3319. (https://doi. org/10.1182/blood-2014-06-580084)

31 Liu G, Xu J, Wu H, Sun D, Zhang X, Zhu X, Magez S \& Shi M. IL-27 signaling is crucial for survival of mice infected with African trypanosomes via preventing lethal effects of CD4+ T cells and IFNgamma. PLOS Pathogens 201511 e1005065. (https://doi.org/10.1371/ journal.ppat.1005065)

32 Wang H, Li Z, Yang B, Yu S \& Wu C. IL-27 suppresses the production of IL-22 in human CD4(+) T cells by inducing the expression of SOCS1. Immunology Letters 2013152 96-103. (https://doi. org/10.1016/j.imlet.2013.05.001) https://ec.bioscientifica.com https://doi.org/10.1530/EC-18-0370 (c) 2019 The authors Published by Bioscientifica Ltd

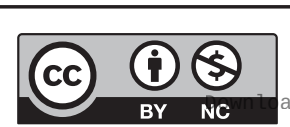

This work is licensed under a Creative Commons Attribution-NonCommercial 4.0 International License. ded from Bioscientifica.com at 04/26/2023 11:15:56AM 
33 Chen Y, Zeng J, Zhang R, Zeng L, Li Y, Wei H \& Yang Q. Effect of interleukin-27 genetic variants on atrial fibrillation susceptibility. Genetic Testing and Molecular Biomarkers 201721 97-101. (https://doi. org/10.1089/gtmb.2016.0219)

34 Ke X, Shen Y, Hu X, Yuan XD, Kang HY, Wang XQ \& Hong SL. [Association between IL-27 gene polymorphisms and susceptibility to allergic rhinitis]. Lin Chuang Er Bi Yan Hou Tou Jing Wai Ke za Zhi 201630 684-688. (https://doi.org/10.13201/j.issn.10011781.2016.09.004)

35 Chen P, Gong Y, Pu Y, Wang Y, Zhou B, Song Y, Wang T \& Zhang L. Association between polymorphisms in IL-27 gene and preeclampsia. Placenta 201637 61-64. (https://doi.org/10.1016/j. placenta.2015.11.003)

36 Pu Y, Chen P, Zhou B, Zhang P, Wang Y, Song Y \& Zhang L. Association between polymorphisms in IL27 gene and renal cell carcinoma. Biomarkers 201520 202-205. (https://doi.org/10.3109/13 54750X.2015.1062555)

37 Horie I, Kawasaki E, Ando T, Kuwahara H, Abiru N, Usa T, Yamasaki H, Ejima E \& Kawakami A. Clinical and genetic characteristics of autoimmune polyglandular syndrome type 3 variant in the Japanese population. Journal of Clinical Endocrinology and Metabolism 201297 E1043-E1050. (https://doi.org/10.1210/ jc.2011-3109)

38 Wemeau JL, Proust-Lemoine E, Ryndak A \& Vanhove L. Thyroid autoimmunity and polyglandular endocrine syndromes. Hormones 201312 39-45. (https://doi.org/10.1007/BF03401285)

39 Zhang S, Gao X, Wang Y, Jia J, Zhang Q \& Ji Z. Interleukin 27 -964A $>\mathrm{G}$ genetic polymorphism and serum IL-27p28 levels in Chinese patients with papillary thyroid cancer. Tumor Biology 201536 8207-8211. (https://doi.org/10.1007/s13277-015-3570-4)

40 Liu L, Wu HQ, Wang Q, Zhu YF, Zhang W, Guan LJ \& Zhang JA. Association between thyroid stimulating hormone receptor gene intron polymorphisms and autoimmune thyroid disease in a Chinese Han population. Endocrine Journal 201259 717-723. (https://doi. org/10.1507/endocrj.EJ12-0024)

41 Yan N, Yu YL, Yang J, Qin Q, Zhu YF, Wang X, Song RH \& Zhang JA. Association of interleukin-17A and -17F gene singlenucleotide polymorphisms with autoimmune thyroid diseases. Autoimmunity 201245 533-539. (https://doi.org/10.3109/0891693 4.2012.702814)

42 Kotkowska A, Sewerynek E, Domanska D, Pastuszak-Lewandoska D \& Brzezianska E. Single nucleotide polymorphisms in the STAT3 gene influence AITD susceptibility, thyroid autoantibody levels, and IL6 and IL17 secretion. Cellular and Molecular Biology Letters 201520 88-101. (https://doi.org/10.1515/cmble-2015-0004)

43 Gaffen SL, Jain R, Garg AV \& Cua DJ. The IL-23-IL-17 immune axis: from mechanisms to therapeutic testing. Nature Reviews Immunology 201414 585-600. (https://doi.org/10.1038/nri3707)

44 Poomarimuthu M, Elango S, Solomon PR, Soundrapandian S \& Mariakuttikan J. Association of IL17 and IL23R gene polymorphisms with rheumatic heart disease in South Indian population. Immunological Investigations 201847 754-764. (https://doi.org/10.108 0/08820139.2018.1493053)

45 Sode J, Bank S, Vogel U, Andersen PS, Sorensen SB, Bojesen AB, Andersen MR, Brandslund I, Dessau RB, Hoffmann HJ, et al. Genetically determined high activities of the TNF-alpha, IL23/ IL17, and NFkB pathways were associated with increased risk of ankylosing spondylitis. BMC Medical Genetics 201819 165. (https:// doi.org/10.1186/s12881-018-0680-z)

46 Yu P, Shen F, Zhang X, Cao R, Zhao X, Liu P, Tu H, Yang X, Shi R \& Zhang H. Association of single nucleotide polymorphisms of IL23R and IL17 with ulcerative colitis risk in a Chinese Han population. PLOS ONE 20127 e44380. (https://doi.org/10.1371/journal. pone.0044380)

Received in final form 10 December 2018

Accepted 29 January 2019

Accepted Preprint published online 29 January 2019
This work is licensed under a Creative Commons Attribution-NonCommercial 4.0 International License. ded from Bioscientifica.com at 04/26/2023 11:15:56AM 